\title{
Interactive comment on "Mineralization of organic matter in boreal lake sediments: Rates, pathways and nature of the fermenting substrates" by François Clayer et al.
}

\section{Anonymous Referee \#2}

Received and published: 15 April 2020

The paper addresses an interesting and fundamentally important question: which fraction of sedimentary organic matter is mineralized through methanogenesis. Based on modeling and analyses of data from two lakes, it argues that organic carbon in negative oxidation states is used preferentially and the hydrogenotrophic pathway of methanogenesis dominates. If true, this may have profound implications for modeling the carbon cycle and interpretations of sedimentary signatures of carbon isotopes. Both the dataset and the model go well beyond the level of detail of typical diagenetic studies, which is indeed a requirement for figuring out the important fine details of organic matter mineralization.

Printer-friendly version

Discussion paper 
This important work, however, could be improved in several key areas.

Style and clarity: The clarity of the narrative deteriorates towards the end of the manuscript. In particular, stating clearly and emphasizing throughout the text the main finding of the work would greatly improve readability. Inferences from modeling of the isotopic profiles could also benefit from a clearer presentation. Key statement such as (Line 265) "practically all $\mathrm{CH} 4$ is produced through hydrogenotrophy" are inferred from modeling d13C profiles, but I admit I was rather lost following the description, particularly trying to separate the relative contributions of hydrogenotrophic vs acetoclastic methanogenesis.

Originality: Much of the work is an update on the results of Clayer et al. 2018. The text should clearly distinguish the novel aspects, especially how (or if) the difference in conclusions is more than just refinement of the numbers from that previous work. For example, a statement on lines 58-60 reads: "Based on the observation that methanogenesis produced $\mathrm{CH} 4$ three times faster than $\mathrm{CO} 2$.... Clayer et al. (2018) concluded that the fermenting OM had a markedly negative COS value of -1.9 ". This parallels the statement in the Abstract, which presumably should highlight the results from this work: "we calculate, from $\mathrm{CH} 4$ and DIC production rates...COS below -0.9". This seems to convey the same information.

Justifying the inclusion or omission of processes: The coupling with the sulfur cycle seems particularly suspect. The cryptic oxidation of sulfide coupled to iron oxides is used as an important pathway for $\mathrm{H} 2$ production. While this reaction is commonly considered (but can be written in various stoichiometries), it is rarely the only reaction that is considered from the complicated network of reactions that comprise the sedimentary Fe and S cycling. Puzzlingly, the modeled SO4 and Fe profiles are not shown (line235). These absolutely need to be shown. The sulfur cycle in this system seems highly unusual. For example (Line 201 and Fig. 2), "SO42- concentrations reach a minimum between SWI and $5 \mathrm{~cm}$ depth, and increase below". These highly unusual features need to be discussed. How can SO4 be produced in anoxic sediment? Does oxida-

Printer-friendly version

Discussion paper
Interactive comment 
tion of $\mathrm{H} 2 \mathrm{~S}$ by $\mathrm{Fe}(\mathrm{III})$ somehow proceed faster than sulfate reduction? What about precipitation of iron sulfides? Similarly, precipitation of $\mathrm{CaCO} 3$ does not seem to be considered as a CO2 sink, while Line 380 mentions that it had to be considered by the used datasets. Were the saturation indexes negative for the study sites?

Discussing implications: If the organic matter used in methanogenesis had negative COS, what happened to the rest of the $\mathrm{C}$ pool? Is oxidized OM not mineralized? Or is it mineralized preferentially earlier, in the water column? What are the implications, e.g. for burial, signature of OM in rock record, etc.? The statement on line 450 seems to address it somewhat, but the statement is not clear. It would also help to discuss how special or typical these lakes are, given that the implications seem to include global extrapolations. For example, diagenesis in Lake Tantare (or is it Lake Bedard? - see below) seems to lack contributions from terminal electron acceptors. How different would this be from a "typical" boreal forest lake?

Other criticisms and suggestions:

Conclusions: "fermentation and methanogenesis represent. . $100 \%$ of OM mineralization ... in Lake Tantare" - Methanogenesis can be fermentation. More importantly, why are there no contributions from terminal electron acceptors? Is it really $100 \%$ ? Confusingly, Fig. 2 shows that sulfate reduction is clearly active in Lake Tantare, whereas contributions of terminal electron acceptors are likely smaller in Lake Bedard.

One of the main results seems to be expressed by Eq. 15. Given the range of COS values $(-1.4+-0.3)$, it might be helpful to state the range in the stoichiometric coefficients explicitly.

Line 284: "i) when labile OM is depleted, ii) with increasing sediment depth" - aren't these two statements in practice the same?

Printer-friendly version

Line 454: "misestimating $\mathrm{CH} 4$ and CO4 production" - not sure what this means. Underestimating the amounts? But early diagenetic models generally work okay and can 
reproduce measured profiles. Are the differences small enough that they are within uncertainties?

Interactive comment on Biogeosciences Discuss., https://doi.org/10.5194/bg-2020-5, 2020. 